MATHEMATICAL ASSOCIATION

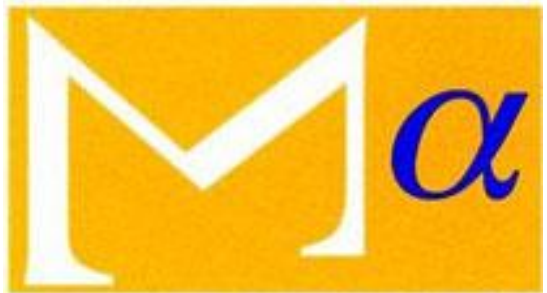

supporting mathematics in education

The Theory of Order, as Defined by Boundaries (Continued)

Author(s): E. T. Dixon

Source: The Mathematical Gazette, Vol. 6, No. 95 (Dec., 1911), pp. 172-178

Published by: Mathematical Association

Stable URL: http://www.jstor.org/stable/3602845

Accessed: 10-03-2016 05:02 UTC

Your use of the JSTOR archive indicates your acceptance of the Terms \& Conditions of Use, available at http://www.jstor.org/page/ info/about/policies/terms.jsp

JSTOR is a not-for-profit service that helps scholars, researchers, and students discover, use, and build upon a wide range of content in a trusted digital archive. We use information technology and tools to increase productivity and facilitate new forms of scholarship. For more information about JSTOR, please contact support@jstor.org. 
that in some mysterious way either the knowledge or the "discipline" does the pupil good, and that "interest" must be mixed with them as jam is mixed with the powder-and for the same reason.

'I'he doctrine that school mathematics is to be an activity reproducing the essential features of historic mathematics is obviously not a "soft" doctrine. For hard and persistent effort as well as exact thinking are certainly among the essential features. But they are characteristics which are not imposed externally; they are the natural marks of intellectual activity when it pursues an end genuinely desired. It would be unreasonable to expect complete parallelism here between the boy and the mathematician. The mathematician selects his own goal and seeks it how he pleases; the boy much accept the choice of his teacher and must work under minutely prescribed conditions of time and place. Apart from his immaturity these differences imply that the internal forces must be supplemented by external influences. It remains true, however, that the boy's efforts are valuable to him only in proportion as they are spontaneous - that is, are the expression of a genuine impulse awakened by an object that really appeals to him.

Misunderstanding on these matters is fatally easy. At the risk of tedium it must be explained that spontaneity does not necessarily mean invention. I show spontaneity in reciting one of Wordsworth's sonnets or in playing a nocturne by Chopin if only I "put myself into it." That is, I can use the words of the poet or the musician's melodies as a means of self-expression. This observation has an important bearing on the question of "mental discipline." The discipline involved in learning mathematics is, ultimately, the boy's attempt to improve his powers by assimilating the methods of the great performers. It is the 'prentice's striving after the touch of the mastercraftsman. In due measure this "formal "element must always be present in an activity which seeks to reproduce actual mathematics. But it must be present as an expression of spontaneity. The purposeless manipulation of symbols has no claim to disciplinary value.

\$9. Summary. Finally it should be urged that the view of algebra teaching bere defended is not only sound in itself, but also includes what is sound in views which, as they are commonly held, must be rejected. Thus, on the one hand, it gives due place to the formal or "disciplinary" elements in mathematics; for these are essential features of the historic activity. And, on the other hand, it guarantees the usefulness of the instruction to those who are to enter mathematical professions. For algebra in the utilitarian stage is to be presented not as a gymnastic manipulation of symbols but as an attempt to understand, and so (in due measure) to control the phenomena of nature and society. Taught in this way, the young engineer should not so often find it difficult to see the connexion between the symbolism of his text-book and the behaviour of his machines. The mathematical specialist should not grow into the state of the Senior Wranglers described by Sir J. J. Thomson, who on entering a physical laboratory "are astonished to find that their formulae are true"; while the general body of boys and girls, who will not make direct applications of their mathematical studies in after-life, should cease to regard algebra merely as a "subject" imposed upon them by the caprice of teachers or examiners, and should gain some appreciation of the part it plays in forwarding important human concerns.

T. P. NunN.

\section{THE THEORY OF ORDER, AS DEFINED BY BOUNDARIES.}

\section{Unique Collations.}

So much for preliminaries. We come now to the formulation of a postulate which may be compared to Euclid's "Let it be granted that 
a straight line can be drawn from any point to any other point"; a postulate, by the way, whose significance has been much underrated by later geometricians. To explain fully the closeness of the analogy would involve the discussion of the cataloguing of groups higher than the first, for which I have not space here, but I may indicate its general nature, to those familiar with the subject, thus: In "projective" geometry the existence of straight lines is assumed, and from them, by means of the Quadrilateral Construction, we obtain an unique method of determining any number of points in a straight line, with reference to three given points in it. My procedure is the exact converse of this. I assume what corresponds to an unique method of determining any number of points in a straight line, with reference to any three of them, and from that $I$ deduce a method of drawing other straight lines, that is, if I am given a plane to draw them in. For this is worth noting: The old method of determining points in a line requires a plane in which to do your Quadrilateral Construction. Hilbert has shown that even that is not enough, and that in order to be able to demonstrate certain of the theorems of projective geometry (notably Desargues' Theorem) from what he lays down as the Axioms of Order, without his Axioms of Congruence, not only a plane, but a three-dimensional space is required.

So far, therefore, the comparison is not to the disadvantage of my methods. But before I have done I shall carry the matter a stage further. The postulate which I assume at this stage, the analogue of Euclid's first postulate, is that we have some unique method of determining a collation in the catalogue, if the collations of any three pairs of units are given, or that if we are given the new names of any three units, we can uniquely determine those of all the rest. Later on I shall show that this can actually be done; and that by pure logic, without any objective, or even conceptual reference to Space.

We have seen that we are free arbitrarily to collate any three units in a group of the first order with any other three, or in a collation in the catalogue, arbitrarily to re-name any three units with any three names; and that it is only when we attempt to collate, or to re-name more than three units that the order of the units in the group imposes any restrictions upon our freedom of choice. But we are not bound to avail ourselves of our full freedom, we may specially consider a restricted class of collations which shall be completely determined by naming three pairs of collated units, or three new names in the places of three old ones. Such restricted collations I call unique collations, a term which may be implicitly defined by the two formal assertions-

(a) In an unique collation, or re-naming, the collation, or new name, of every unit of a group of the first order may be determined from the collation, or new name, of any three of them.

(b) The resultant of two unique collations is itself an unique collation; that is to say, if we represent a number of successive collations by a scheme of a number of lines, each being uniquely collated with the next, then the collation of the first and third, or generally of any two lines of the scheme, will be unique.

Now let us deternine an unique collation by arbitrarily collating three names, say $C_{1}, K_{1}$, and $M_{1}$, with three others; which, to avoid implications as to their order, I will represent by small letters, say, $p_{2}, q_{2}$, and $r_{2}$, respectively.

If $p, q$, and $r$ represent three different names, we may now make another unique collation, collating $p_{2}, q_{2}, r_{2}$, with $C_{3}, K_{3}, M_{3}$, respectively; and again we may collate $C_{3}, K_{3}, M_{3}$, with $p_{4}, q_{4}, r_{4}$, uniquely.

Then by clause (b) of the definition it follows that the collations (1)-(3) and (1)-(4) and (2)-(4) are all unique. 
But the collations (1)-(2) and (1)-(4) may both be determined by collating $C, K, M$, with $p, q, r$, respectively, and therefore by clause $(a)$ they must be one and the same collation; that is, in collation (2)-(4) every name is collated with itself. And as such a collation is determined by collating any three units, it follows that:

(12) If thrce units are collated identically, then every unit is collated identically; and this particular unique collation may be called The identical collation.

Now in (1) and (3) we have $C, K, M$, each collated identically. Hence this also is the identical collation.

(13) It follows that: If a collation (1)-(2) is unique, so is the collation (2)-(1).

It may be shown that all unique collations are either positive, negative, or zero collations, that is, that the only discontinuous unique collations are those in which one unit in each group is collated with all the units of the other group; in the case of collations in the catalogue, zero unique collations are, or are not, reciprocal, according as it is the same, or two different units which are so collated.

We may classify unique collations in the following different ways-

Firstly, they may be positive, negative, or zero collations.

Secondly, they may be reciprocal collations, or not.

Thirdly, we can make a classification by the number of units which are collated identically, or we may say, the number of names unchanged by the collation. In this classification we shall have-.

(1) More than two units collated identically.

There is, however, only one collation of this class, namely, The Identical collation. It is obviously a positive collation. (Class 3.)

(2) Two, and only two, units collated identically. All negative collations come under this head. (Class 2a.) There are also positive collations. (Class 2b.)

Zero collations, if not reciprocal, are all in this class also. (Class 2c.)

(3) One, and only one, unit is collated identically. These may be positive. (Class 1 a.) Or zero, if reciprocal. (Class $1 b$.)

(4) No unit is collated identically. These are all positive. (Class 0.)

(14) The proposition which I am about to prove, viz.: If in any unique collation any one pair of units is collated reciprocally, then every pair is collated reciprocally, and it may be called a reciprocal collation, is a very important one, and for this reason alone it would be worth while giving the proof at some length. I do so also to illustrate the methods of reasoning, and the use of schemes; giving first a proof in ordinary language, and then the same by means of a scheme.

Let us consider any four names in the catalogue, let us say $C, F, L$, and $Q$. Of these two pairs, $(C L)$ and $F Q$ ) separate one another, while the other two pairs of pairs do not. We have then three cases to consider :

(i) we may obtain a negative collation by collating reciprocally $C$ and $F$;

(ii) we may obtain a positive collation by collating reciprocally $C$ and $L$;

(iii) we may obtain a zero collation. I will take these cases separately.

(i) Let us collate $C$ and $F$ reciprocally, and $L_{1}$ with $Q_{2}$. We may pass the collation under review so that from $C_{1}$ we pass to $Q_{1}$ and $L_{1}$ before reaching $F_{1}$. Therefore we also pass from $F_{2}$ to $Q_{2}$ before reaching $C_{2}$. The collation is a negative one, and there must be two names, which we may represent by $h$ and $k$, which are collated identically.

We might therefore define the collation sufficiently by saying that 
$h_{1}, C_{1}, k_{1}$ are collated respectively with $h_{2}, C_{2}, k_{2}$, and from this it would follow also that $C_{1}$ was collated with $F_{2}$, and $L_{1}$ with $Q_{2}$.

Now let us repeat the collation, collating $h_{2}, F_{2}, k_{2}$, with $h_{3}, C_{3}, k_{3}$, respectively. It must follow, as before, that $C_{2}^{\prime}$ is collated with $F_{3}$, and $L_{2}$ with $Q_{3}$.

And now, if we pass in review all three names at once, we see that $h_{1}, F_{1}, k_{1}$ are collated respectively with $h_{3}, F_{3}, k_{3}$, and since the collations are unique, the collation (1)-(3) is the identical collation. Therefore $Q_{1}$ and $Q_{3}$ are collated together; and since $L$ was collated with $Q$, it is collated with $Q$ also. That is, $Q$ and $L$ are collated reciprocally by (1)-(2), as well as $C$ and $F$. And what is true of $Q$ and $L$ is true of every other collated pair of units.

This argument may be exhibited by the following scheme:

$$
\left.\begin{array}{ccccccc}
(1) & C & h & F & L & k & (Q) \\
(2) & F & (h) & C & Q & (k) & L \\
(3) & C & h & (F) & (L) & k & (Q)
\end{array}\right\}
$$

In line (1) the letters are written in any order, though it looks better to write them in their real order if we know it. $Q$, however, is not written in at once, as the brackets show its collation with line (2) will be determined later. In line (2) we write $F, C$, and $Q$ under $C, F$, and $Q$ in line (1); $h$ and $k$ represent identically collated units, therefore we write $h$ under $h$, and $k$ under $k ; L_{2}$ is written in anywhere. In line (3) we write $h, C, k$ under $h, F, k$, respectively in line (2). Then we can copy the collation (1)-(2), and so write in $\left(F_{3}\right),\left(Q_{3}\right)$ under $C$ and $L$ in line (2). And since the collation (1)-(3) is seen to be identical, we can write in $(Q)$ in line (1) over $L$ in line $(2)$, and $(Q)$ in line (3). It is now obvious that the collation of $Q$ and $L$ in lines (1) and (2) is reciprocal.

Similarly, if we collate $F$ and $L$ reciprocally, the collation being also negative, if $C_{1}$ is collated with $Q_{2}$, then $Q_{1}$ will be collated with $C_{2}$. But if we collate $C$ and $L$ reciprocally, the collation is positive, and the above argument does not apply. The proof in this case is as follows:

(ii) Let us pass in review three names at once. When the first is $C$ let the second be $F$, and the third $L$. When the first is $F$ let the second be $C$, and the third $Q$. When the first is $L$ let the second be $Q$, and the third $C$. That is, the collation (1)-(2) collates $C$ and $F$ reciprocally, and $L_{1}$ with $Q_{2}$ : consequently also $Q_{1}$ with $L_{2}$. The collation $(2)-(3)$ collates $C$ and $Q$ reciprocally, and $F_{1}$ with $L_{2}$ : consequently also $L_{1}$ with $F_{2}$.

It follows that the collation (1)-(3) collates $C$ and $L$ reciprocally, and also $F$ and $Q$ reciprocally; and since this is a possible unique collation, it is the only one which collates $C$ and $L$ reciprocally, and $F_{1}$ with $Q_{3}$.

This argument is illustrated by the following scheme:

$$
\left.\begin{array}{ccccccccc}
(1) & C & h & F & & L & k & Q & \\
(2) & F & (h) & C & r & Q & (k) & (L) & s \\
(3) & L & & Q & (r) & C & & (F) & (s)
\end{array}\right\}
$$

Under $C, F, L$, in line (1) we write $F, C, Q$, in line (2), and $L, Q, C$, in line (3). The identically collated names represented by $h, k$ in lines (1) and (2), and by $r, s$ in lines (2) and (3), indicate that the collations (1)-(2) and (2)-(3) are both negative. Consequently, we may write in $(L)$ in line (2), and $(F)$ in line (3), under $Q$ in line (1). On inspection we see that the collation (1)-(3) is reciprocal.

(iii) There remains the case of a zero collation. If in such a collation two units are collated reciprocally, say $C$ and $L$, one of them, say $C_{1}$, is collated also with all the other names in the catalogue, amongst them therefore with $C_{2}$. But by hypothesis, $C_{2}$ was collated with $L_{1}$. Therefore $C_{2}$ is 
collated discontinuously, and with every other unit in (1). Every collation is therefore reciprocal.

We may therefore determine a reciprocal unique collation by naming any two pairs of units which are to be reciprocally collated. Such a change in the catalogue may be called an inversion; we may be said to have inverted the catalogue with respect to the two pairs. I find it convenient further to distinguish between positive inversions, which I call obversions, and negative ones, reversions.

We may also determine inversions in other ways, which differ according as they are negative, zero, or positive. These I proceed to describe.

(15) A reversion may be determined by naming its two self-conjugate units.

For though only two units have been named, which at first sight would seem insufficient to determine any unique collation, we are in reality given an additional datum, namely, that the collation is to be a reciprocal one. To show that this is enough, consider the scheme-

$$
\left.\begin{array}{lcccc}
\text { (1) } & C & h & (x) & k \\
(2) & (x) & h & C & k \\
(3) & (x) & k & C & h
\end{array}\right\}
$$

In lines (1) and (2) we represent a reciprocal collation in which two names, $h$ and $k$, are identically collated. But, only two given collations, viz. : $h$ with $h$ and $k$ with $k$, are shown in the scheme, since $(x)$ has not yet been determined (as is indicated by the brackets), and we do not therefore appear to have the means of determining an unique collation. But, to find $x$, let us add a third line to the scheme, by inverting (1) with respect to the pairs $(C x)$ and $(h k)$. We see now that in (2)-(3) we have three given collations, viz.: $h$ with $k, C$ with $C$, and $k$ with $h$. Thus the collation (2)-(3) is uniquely determined. And we may go back and begin over again, by writing lines (2) and (3) of the scheme down first. They represent a reciprocal collation, since $h, k$ are reciprocally collated. And since $C$ is identically collated (and is not collated with either $h$ or $k$ ), it is a negative collation, and therefore some other unit, separated from $C$ by $(h k)$, is also identically collated. This unit, represented by $x$ in the scheme, is therefore uniquely determined; and so we are in a position uniquely to determine the collation (1)-(2).

The case of an obversion is, however, not quite so simple, for in such a collation no one unit or pair of conjugate units is marked out in any way from any other.

Consider again for a moment the above scheme $(d)$. Lines (1)-(2) represent a reversion with respect to $(h k)$, in which $(C x)$ is a conjugate pair. Lines (2)-(3) represent also a reversion, with respect to $(C x)$, one of the conjugate pairs of the former reversion. And in this case $(h k)$ is a conjugate pair. Thus it follows that:

(16) If we revert with respect to a conjugate pair of a reversion, we interchange its self-conjugate units, which form therefore a conjugate pair of the second reversion.

Let us see what happens if we revert with respect to a conjugate pair of an obversion. The conjugate pairs of the obversion all separate one another, while no two of the conjugate pairs of the reversion do so. Consequently there cannot be more than one conjugate pair common to both. It can be shown that there always is one, thus: if we pass in review the cenjugate pairs of the reversion starting from one of the self-conjugate units, $h$, to the other, $k$; initially the conjugate pair under review, say $\left(x x^{\prime}\right)$, separates $h$ from the conjugate of $x$ in the obversion $x^{\prime \prime}$, for initially $x^{\prime \prime}$ is at $k$. But finally $x^{\prime \prime}$ is at $h$, and therefore it passes the boundary $\left(x x^{\prime}\right)$, and as $x^{\prime \prime}$ can never be $x$, it must have 
been $x^{\prime}$. Hence in a given obversion any given unit determines, first a second unit, its conjugate, and secondly a conjugate pair, which belongs also to the reversion with respect to the first-named pair of units. A pair of pairs of units so situated with respect to an obversion may be called a quartette of the obversion, and we may say that:

(17) In an obversion each unit determines a quartette. Since a quartette consists of two pairs of units which intersect one another, it is obvious that, conversely, a quartette is enough to determine an obversion.

(18) We see now, however, that: An obversion may be determined by naming a conjugate pair and one more unit of a quartette, for the fourth can be determined by reverting the third with respect to the other two. It will be noted that this does not amount to as much as naming three collations; for the only collations named are the two reciprocal collations of the conjugate pair.

In the last case, that of the zero inversion, it is obvious that to name the one self-conjugate unit is all that is needed to determine the collation. But the significance of this fact, or indeed of zero collations at all, depends, in a manner familiar to mathematicians, entirely on their being limiting cases of positive or negative collations. In order to understand this, let us consider the reciprocal collations determined by the conjugate pairs $(C F)$ and $(L q)$, where $q$ represents a name, initially $Q$, which we may pass in review round the catalogue. We thus pass in review a group of inversions, and collate them with the letters of the catalogue. They form, therefore, an uniform group of the first order. Initially the inversion is a reversion, for $(C F)$ and $(L Q)$ do not separate one another. There will, therefore, be two self-conjugate units, say $h, k$; of which one, say $k$, is separated by $(L q)$ from all the other named units. If $q$ passes to $L$, as it reaches $L$ both it and $k$ come to mean $L$, which is a self-conjugate unit. As $q$ passes on, $(L q)$ again separates $k$ from the other named units. Now the boundary $(h k)$ constantly divides the whole catalogue into two parts, one contains $F$ and $L$, the other $C$ and $q$, and the conjugates of all the units in the other part. Hence, when $q$ comes to mean $C$, and therefore we have a zero collation, $C$, and $C$ alone, is the conjugate of all the units in the other part. Therefore the boundary $(h k)$ must consist of the two units contiguous to $C$, i.e. $h$ and $k$ are no longer distinguishable from $C$, which, besides being conjugate to all the other units, is both self-conjugate units rolled into one. As soon as $q$ has passed $C$, the inversion becomes an obversion, and continues so till it reaches $F$, when it again passes through the limiting case of a zero inversion, and reappears as a reversion.

(19) It is obvious that two successive negative collations must effect a positive one. We have already seen this in scheme $(c)$. In particular: If in an obversion, we revert twice successively with respect to the conjugate pairs of a quartette, we effect the obversion itself. For consider the following scheme, where the conjugate of any letter with respect to the obversion is represented by the letter with a double accent.

$$
\left.\begin{array}{lllllllll}
\text { (1) } & (a) & h & (b) & k & \left(a^{\prime \prime}\right) & h^{\prime \prime} & \left(b^{\prime \prime}\right) & k^{\prime \prime} \\
\text { (2) } & (b) & h & (a) & k^{\prime \prime} & \left(b^{\prime \prime}\right) & h^{\prime \prime} & \left(a^{\prime \prime}\right) & k \\
(3) & \left(a^{\prime \prime}\right) & h^{\prime \prime} & \left(b^{\prime \prime}\right) & k^{\prime \prime} & (a) & h & (b) & k
\end{array}\right\}
$$

Here $\left(h h^{\prime \prime}\right)$ and $\left(k k^{\prime \prime}\right)$ form a quartette of the obversion, (1)-(2) is a reversion with respect to $\left(h h^{\prime \prime}\right)$, and (2)-(3) one with respect to $\left(k k^{\prime \prime}\right)$. And evidently (1)-(3) is the obversion itself. But note also that if $a$ represents any name, which reverts into $b$ with respect to $\left(h h^{\prime \prime}\right)$, then the unit collated in the third line with $a$ and $b$ must be called $a^{\prime \prime}$, in virtue of the collation (1)-(3), which is the obversion. We may 
similarly write $a$ in line (3) under $a^{\prime \prime}$ in line (1), and so on, completing line (3). As to line (2), since (1)-(2) is reciprocal, and we have $b$ under $a$, we have also $a$ under $b$, and similarly with the other letters, between brackets.

(20) Looking now again at the first two lines of the above scheme, we see that: Reversion with respect to a conjugate pair of any inversion does not alter the conjugate relation. For example, reversion with respect to $\left(h h^{\prime \prime}\right)$ changes $a$ into $b$, but it also changes the conjugate of $a$ in the obversion, $a^{\prime \prime}$, into $b^{\prime \prime}$, its conjugate in the same inversion. In the case of a negative inversion, a reversion with respect to one of its conjugate pairs merely interchanges the self-conjugate units, in the case of a zero inversion it does not alter the self-conjugate unit, so that in these cases also the conjugate relation is unaltered.

It is worth while adding the enunciations of certain theorems about successive reversions, though, as they do not lie in the direct line of my argument, I shall only add the proof of one of them, which happens to be of special interest.

(21) Any positive unique collation may be effected by successive reversions with respect to two pairs of units.

If the pairs do not separate one another, the collation is of class 0 .

If the pairs have a common unit, it is of class 1a.

If they separate one another, it is of class $2 \mathrm{~b}$.

If they have two common units, it is of class 3 (the identical collation).

The proof of the first proposition is as follows:

In the scheme

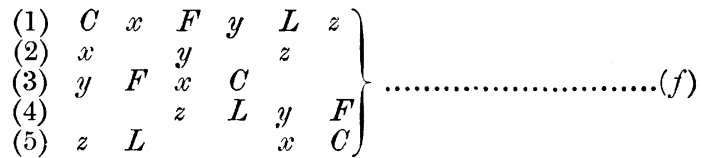

If we choose any three catalogue names whatever, say $C, F$, and $L$, and collate them with any three other names, represented by $x, y$, and $z$, then (1)-(2) may represent any unique collation whatever. Invert (1) with respect to $(C y),(F x)$ to make $(3)$, with respect to $(F z),(L y)$ to make (4), and with respect to $(L x),\left(C_{z} z\right)$ to make (5).

It is evident by inspection that the collations of line (2) with each of the subsequent lines are reciprocal. Thus any unique collation, (1)-(3), may be effected by two successive reciprocal collations; e.g. (1)-(2) and (3)-(2). It remains to be proved that if (1)-(2) is positive, the two reciprocal collations may be so chosen as to be both negative.

It will be sufficient to prove that one of them may be chosen negative; since the other must in that case be the same, in order to result in a positive collation. I shall therefore show that at least one of the collations of line (1) with (3), (4), or (5) must be negative.

The condition that the collation (1)-(3) should be positive is that $(C y)$ should separate $(F x)$. But, the collation (1)-(2) being positive (see Theor. 7), this is also the condition that in this collation the collated pairs $\left(C_{1} x_{2}\right)$ and $\left(F_{1} y_{2}\right)$ should be separated by two identically collated pairs, $\left(p_{1} p_{2}\right)$ and $\left(q_{1} q_{2}\right)$, say. Similarly, if the collation (1)-(4) is positive, then $\left(p_{1} p_{2}\right)$ and $\left(q_{1} q_{2}\right)$ also separate $\left(F_{1} y_{2}\right)\left(L_{1} z_{2}\right)$. But if both these conditions hold, we cannot possibly also have $\left(p_{1} p_{2}\right)$ and $\left(q_{1} q_{2}\right)$ separating $\left(L_{1} z_{2}\right)\left(C_{1} x_{2}\right)$, and therefore $(C z)$ cannot separate $(L x)$, and the collation (1)-(5) must be negative. That is, the positive collation (1)-(2) may be effected by the two reversions (1)-(5) and (5)-(2).

( $T$ o be continued.)

E. T. Dixon. 